\title{
LEAST SQUARES NULL SPACE VARIATIONAL CBARACTERIZATION FOR NONMINIMUM NORM SOLUTIONS
}

\author{
S. Konyk, Jr* , M. G. Amin*, M. G. Lagunas** \\ -Department of Electrical Engineering \\ Villanove Univeraity \\ Villanova, PA 19085, USA
** Proceasdo de Senal en Comunicaciones E.T.S.I. Telecomunicacion \\ Apdo. 30.00208080 Barcelona, Spain
}

\section{ABSTRACT}

The least squares eotimation problem with nonminimum norm conetraints on the unknown model parameter is connidered. Contrary to the quadratic contraint least squares solutions the approach preeented does not neceer sarily satiofy the constraint, but rather relies on the nutlity of the dats matrix to maintain the unconstrained least squares error value while trading off the minimum norm solution by another with the shorteat distance from the null spece of the constraint. The SVD of the data matrix is used to obtain the neceseary information about the minimum norm solution as well as the bacis of the null apace. Closed form expreseions are derived for the case in which the constraint of intereat is the smoothness of the model perameters. Examples of sinusoids in white noise are given for illustration.

\section{INTRODUCTION}

In the least-equares estimation (LSE) problem, the nonsero nullity property of the data matrix may ariee mootly due to the nature of the data, as in the case of noise free sinueoids [1]. This property, however aloo manifeats itself in the noisy environment as a coneequence of procesaing the data prior to extimation to increase the signal-to-noise ratio [2]. In the latter case, the data is projected into the signal subspace and results in a reduced rank expression of the nonsingular date matrix. When constraints other than minimum norm exist on the optimum weight vector the null space of the date matrix can be searched in an attempt to satisfy the constraints without increasing the minimum norm solution error value.

In this paper the above LSE problem is considered in the context of a multiple linear regreseion model implemented by a transversal filter whose tap weights are the unknown model parameters. In this context, caves are considered for which the model parameter estimate is derived from a finite block of data suples whose correaponding data matrix posseseses the nonsero nullity property. An optimal solution to this problem is, therefore, any model parameter vector that yields the minimum poesible value of the sum of error squares performance criterion. This olution charscterises a parameter subepece whose dimension is equal to that of the data matrix nullity.

For the well-known minimum norm constraint, the result is a unique solution determined by the peeudo inverse of the data matrix and corresponds to selecting a particular member of the subepece of the weight vectors which intersect only the origin of the nullspace. The invariant performance impoeed by the span of the null apace is used as a reference in searching for and selecting an optimal LSE solution from a set of competitive suboptimal features.

Searching the nullspace is performed by firnt defining the LSE null space basie via singular value decomposition (SVD) of the dats matrix [3]. These beese are then used to determine the additional null space weight components which when added to the minimum norm solution result in a total weight vector which has the shortest diatance from the null space of the nonlinear constraints.

The paper presents an insightful approach to solve the above type LSE of problems. Detailed analysis is given to the case where amoothness is chosen as the nonlinear constraint of intereat. Smoothnes [4] is a measure of how clooe the filter weights are to a constant mean value. It is viewed as driving the filter towards a rectangular impule response or equivalently a sinc function in the frequency domain. In the $z$ domain, the smoothness constraint translate into equally spaced zeros of unit value excluding that of zero phase.

Two examples of one step forward linear prediction are presented in which smoothness constraints are considered as the quadratic constraints on the predictor weights. The data in both examples consists of two sinusoids with widely spread frequencies in additive gansoian white noise. The firet example represents the noiseless case where the noise power level is set to zero. In this case, with $M \geq 4$, the nonzero nullity of the data matrix is a natural property of the least-squares problem formulation. The nullity in the noisy case, on the other hand, is eatablished through the rank reduction process which involes setting the noise eigenvalues to sero [b]. Both examples show that by moving in the null space of the data matrix, or its reduced rank form, a amoother filter impulee response can be obtained at no expense of the least-aquares error.

\section{NULL SPACE LEAST SQUARES}

The least equares estimation problem is considered in the context of a linear predictor. The one otep predictor output $\hat{u}(i)$ may be expresoed as the convolution oum 


$$
d(i)=\sum_{k=1}^{M} w_{k} u(i-k+1)
$$

where $M$ is the filter length, $w_{k}$ are the filter weights and the $u(\cdot)$ are the tap inputs. Employing the covarience method for windowing the input data $u(i), i=1, \ldots, N$; the sum of error equares measure is given by

$$
J_{\varepsilon}\left(w_{1}, \ldots, w_{\mu}\right)=\sum_{i=M}^{N}|e(i)|^{2}
$$

where

$$
e(i)=u(i)-\hat{u}(i)
$$

Equation (2) may be expreseed as

$$
\begin{aligned}
J_{\varepsilon}(\boldsymbol{w}) & =\mathbf{r}^{T} \mathbf{r} \\
& =(\mathbf{b}-\mathbf{A} \boldsymbol{w})^{T}(\mathbf{b}-\mathbf{A} \boldsymbol{w})
\end{aligned}
$$

where

$$
\begin{aligned}
\boldsymbol{w} & =\left[w_{1}, w_{2}, \ldots, w_{M}\right]^{T}, \\
\mathbf{x} & =[e(M), e(M+1), \ldots, e(N)]^{T}, \\
\mathbf{b} & =[u(M+1), u(M+2), \ldots, u(N+1)]^{T},
\end{aligned}
$$

and

$$
A=\left[\begin{array}{cccc}
u(M) & u(M-1) & \ldots & u(1) \\
u(M+1) & u(M) & \ldots & u(2) \\
\vdots & \vdots & \vdots & \vdots \\
u(N) & u(N-1) & \ldots & u(N-M+1)
\end{array}\right]
$$

The superseript $T$ denotes transposition, $\boldsymbol{W}$ is the $M \times 1$ tap weight vector, $\mathbf{n}(i)$ is the $M \times 1$ input vector, $r$ is the $M \times 1$ reaidual vector, and $A$ is the $(M-N+1) \times N$ date matrix.

The least squares solution of the filter weights, which minimizes $J_{\varepsilon}$ over the data window, satisfies the normal equation

$$
\mathbf{A}^{T} \mathbf{A} \hat{\mathbf{w}}=\mathbf{A}^{T_{b}}
$$

If $\operatorname{rank}(\mathrm{A})=M, A \mathrm{~A}^{\mathrm{T}}$ is nonsingular and the tap weight vector is uniquely determined as $\hat{w}=\left(\mathbf{A} A^{T}\right)^{-1} A^{T} b$. On the other hand, for $\operatorname{rank}(A)=r<M$ the nullity of $A$ is nonzero and the least squares solution, $\hat{\boldsymbol{w}}$, is no longer unique. In this case, the poeudo inverse of the data matrix, denoted $\mathbf{A}^{\mathbf{t}}$, characterizes the minimum norm solution that is given by

$$
\begin{aligned}
\hat{\mathbf{w}} & =\mathbf{A}^{\mathbf{l}} \mathbf{b} \\
& =\mathbf{X} \Sigma^{-1} \mathbf{Y}^{T}
\end{aligned}
$$

The orthogonal trandormation matrice $\mathbf{Y}$ and $\mathbf{X}$ of the singular value decomposition (SVD) of $\mathbf{A}$ characterize the null space, $N(A)$, and the range space, $R(A)$, of the data matrix, respectively. The first $r$ diagonal entries of $\boldsymbol{\Sigma}$ satiofy $\sigma_{1} \geq \sigma_{2} \geq, \ldots, \sigma_{M} \geq 0$ while the rest of the matrix elements have zero values. The last $(M-r)+1$ columns of the matrix $\mathrm{X}$ form an orthonormal basis for $N(A)$ and will be used in the next section to move away from the minimum norm solution toward satiofying the nonlinear constraint.

\section{SMOOTHNESS CONSTRAINT CASE}

The approach to null eppace least aquarea in applied to the case where sonlinear smoothnes constraint is imposed on the weight vector. The amoothneas constraint provides a simple example which illustrates the fundamental mechaniom of null space least squares. The goal here is to constrain the weight vector to the minimum possible smoothness measure which still results in the minimum least squares error. For a given weight vector $w$, a measure of its smoothness is expressed as

$$
\begin{aligned}
J_{0}(w) & =\sum_{i=1}^{M}\left(w_{i}-\bar{w}\right)^{2} \\
& =w^{T} Q w
\end{aligned}
$$

where $\bar{w}$ is the average value of the weights and $Q$ is an $M \times M$ symmetric matrix with entries

$$
q_{i, j}= \begin{cases}\frac{M-1}{M} & \text { for } i=j \\ \frac{-1}{M} & \text { for } i \neq j\end{cases}
$$

The behavior of the smoothnees measure for $M=2$ is illustrated in Figure 1. The null space of $\mathbf{Q}$ is one dimensional and spanned by the $M \times 1$ unit vector

$$
\hat{\mathbf{I}}=\left[\begin{array}{c}
\frac{1}{\sqrt{m}} \\
\vdots \\
\frac{1}{\sqrt{m}}
\end{array}\right]
$$

A maximally flat impulse reeponse corresponds to zero variance in the weight vector which implies perfect smoothness and thus

$$
W=d \text { for some } c \in R
$$

The set of column vectors $\boldsymbol{x}_{\mathbf{r}+1}, \ldots, \boldsymbol{x}_{M}$ of $\mathbf{X}$ form an orthonormal basis for $N(\mathrm{~A})$. Moving a weight vector through $N(\mathbf{A})$ does not change its LSE error, i.e. $J_{\varepsilon}(\mathbf{w})=$ $J_{\varepsilon}(w+\Delta w), \forall \Delta w \in N(\mathcal{A})$. Thus the least squares problem in terms of the bases for $N(\mathrm{~A})$ and $N(\mathrm{Q})$ is: given the minimum norm least equares weight vector $\$$, find the Bcalars $c, \alpha_{r+1}, \ldots, \alpha_{M}$ which minimize

$$
\begin{gathered}
J_{\mathcal{N} E}(\mathbf{w})=\left\|\hat{\mathbb{I}}-\hat{\boldsymbol{w}}_{T O T}\right\|^{2} \\
\hat{\mathbf{w}}_{T O T}=\sum_{i=r+1}^{M} \alpha_{i} \mathrm{x}_{i}
\end{gathered}
$$

It can be shown that the ocalars in (15) which maximize smoothnes without increasing the least equares error are

and

$$
\hat{c}=\frac{\hat{\mathbf{I}}^{T} \hat{\mathbf{w}}}{1-\sum_{i=r+1}^{M} \hat{I}^{T} x_{i}}
$$

$$
\hat{\boldsymbol{\alpha}}_{i}=\hat{\boldsymbol{c}} \mathbf{I} \mathbf{x}_{\boldsymbol{i}}
$$


Two eimulation examplea are given in Section IV which use (15-17) to improve smoothness in the case of sinusoids with additive white noive.

\section{SIMULATIONS}

Twenty dats samples $x(n) \quad n=1, \ldots, 20$ are generated with

$$
x(n)=\cos \left(\omega_{1} n\right)+\cos \left(\omega_{2} n\right)+\sigma w(n)
$$

where $w(n)$ is a white noise nequence whose power level is choven to provide infinite SNR in the noiselese case and $20 d B$ SNR in the noiny case. In both cases, $\omega_{1}=$ $\pi / 3, \quad \omega_{2}=\pi / 6 \mathrm{rad} / \mathrm{sec}$ and the filter length $M=11$.

Naise free case. In this case, the null space of $A, N(A)$, is of dimension 7. The minimum norm solution is $\hat{\boldsymbol{t}}=$ $[0.3415,0.0000,-0.2500,-0.5000,-0.0915,0.0000,-0.0915$, $-0.2500,-0.2500,0.0000,0.3415]^{T}$, which yields a smoothness $\hat{\boldsymbol{w}}^{T} Q \hat{\boldsymbol{w}}=0.4773$ and zero prediction. The zeros of the $z$ domain weight vector polynomial include $\pm e^{j \pi / 8}, \pm e^{j \pi / 0}$ which correspond to the two sinusoidal frequencies. Incorporating $*$ with the last seven columns of the matrix $\mathbf{X}$ in equation (15) results in $\hat{c}=-3.3166$. From (16) and (17), $\hat{\boldsymbol{\omega}}_{\text {TOT }}=[-1, \ldots,-1]^{T}=-\hat{\mathbf{I}}^{\boldsymbol{T}}$. Accordingly $\hat{\boldsymbol{\omega}}^{T} Q \hat{\boldsymbol{t}}=0$, i.e. maximum smoothness (flat response) is obtained while maintaining $J_{E}=0$. As shown in Figure 2, the new set of zeros still include $\pm e^{j \times / 8}, \pm e^{j * / 0}$, i.e., the polynomial roots which correspond to the input signal did not exhibit any displacement as a result of operating in $N(\boldsymbol{A})$. This property is satisfied for any et of values of $\omega_{1}$ and $w_{2}$. Figure 3 shows the weight vector before and after smoothing.

Noisy case. In this case, the SVD of $A$ in first performed and followed by setting the last 7 values of the diagonal et ements of $\Sigma$ to zero. With the new $\Sigma$ the reduced rank form of $A$ is obtained using the same orthogonal matrices $X$ and $Y$. The rank reduction of $A$ provides and an improvement of smoothnese over the full rank original data matrix and reduces $\hat{\boldsymbol{w}}^{T} Q \hat{\boldsymbol{w}}$ from 3.3911 to 0.4954 . The rank reduction however causes an increase in the LSE error from approximately zero to $\mathbf{0 . 2 5 3 3}$. After reducing $r$ from 11 to 4 the problem becomes similar to the noiselese case. The corresponding value of $\dot{c}$ is found to be $-\mathbf{3 . 8 0 8 5}$ and the total weight vector obtained from equation (15) hae kept $J_{\varepsilon}=.2533$ while further reducing the smoothness to 0.0246. Figure 4 shows a plot of the three different values of the optimum weight vector results from the above process. While the weight vector, besed on the original data matrix is highly unsmooth, the nonminimum norm weight vector of the reduced rank date matrix is almont flat. This emoothnese improvement is also shown in Figure 5 which illustrates the immigration of the $z$ domain roots of the weight vector polynomial towards the unit circle.

\section{v. CONCLUSIONS}

The paper presented an insightful approsch to the least squares estimation problem in which 1) the data matrix has nonsero nullity, 2) it is desired to minimize a nonlinear quadratic function in the estimator weight vector.
Contrary to the solution of the constraint least squares problem $[6,7]$ which satiofies the constraint by treding off the unconstrained least squares error value, the weight vector in the underlying problem is set to be as close as poosible to the constraint null space without an increase in the estimation error. The key to this approsch is to define the basis of the null space via the SVD of the data matrix which also provides the minimum norm solution. There bases are then used to drive the weight vector away from its minimum norm value to an optimum solution which maintains the LSE error and has the shortest distance from the null space of the construint. A theoretical frame work is developed with closed form expression for the case where smoothness represents the quadratic function. Verification is given using examples of sinusoid in white noise.

\section{REFERENCES}

[1] S. Marple, Digital Spectral Analysis, Prentice-Hall, Englewood Cliffs, NJ, 1987.

[2] S. Haykin, Adaptive Signal Proceseing, Prentice-Hall, Englewood Cliffs, NJ, 1986.

[3] G. Golub and C. Van Loan, Matrix Computations, Johns Hopkins University Press, 1983.

[4] S. Konyk and M. Amin, "A new clases of nonlinearly constrained linear estimators," IEEE International Conference on Acoustica, Speech and Signal Proces., New York, NY, April, 1988.

[5] D. Tufts and R. Kumaresan, "Estimation of frequencies of multiple vinusoids making linear prediction perform like maximum likelihood," Proceedings of the IEEE, pp 975-989, 1982.

[6] O. Frost, "An algorithm for linearly constrained adaptive array proceseing," Proceedings of the IEEE, August, 1972.

[7] T. Mu and L. Griffiths, "A solution space approsch to achieving partially edaptive arrays," IEEE International Conference on Acoustics, Speech and Signal Procss., New York, NY, April, 1988.

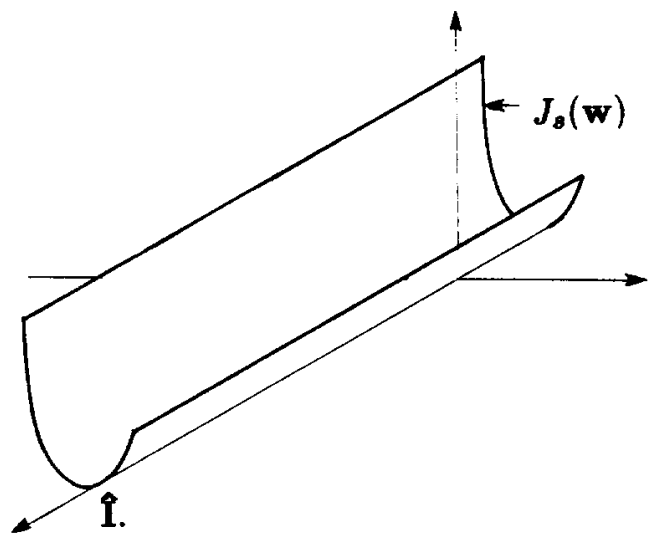

Fig. 1 Illustration of the invariance of the smoothnese measure, $J_{a}(\boldsymbol{w})$, with respect to its null space $\hat{\mathbf{L}}$. 


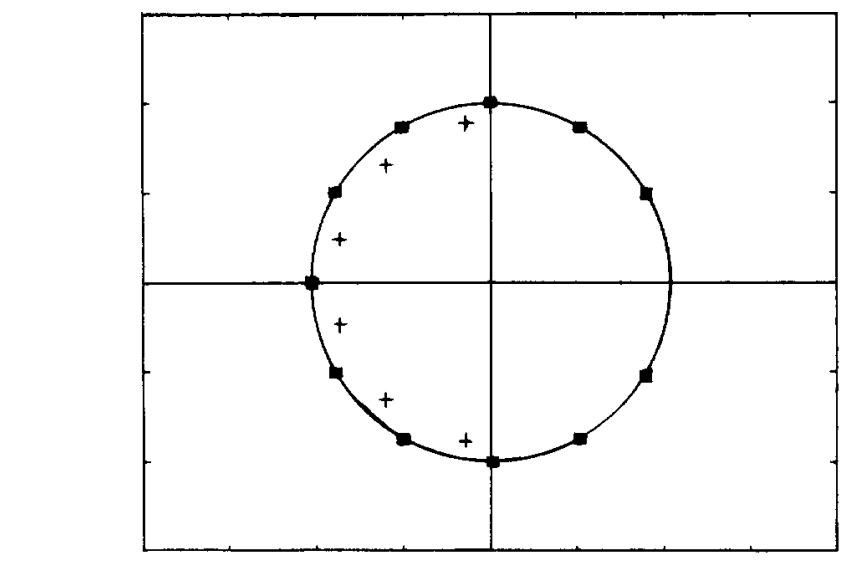

Fig. 2 Zero diagram of the LSE weight vector in the noise free example, $(x)$ for $\hat{w},(\mathbf{m})$ for $\mathbf{w}_{\text {TOT }}$.

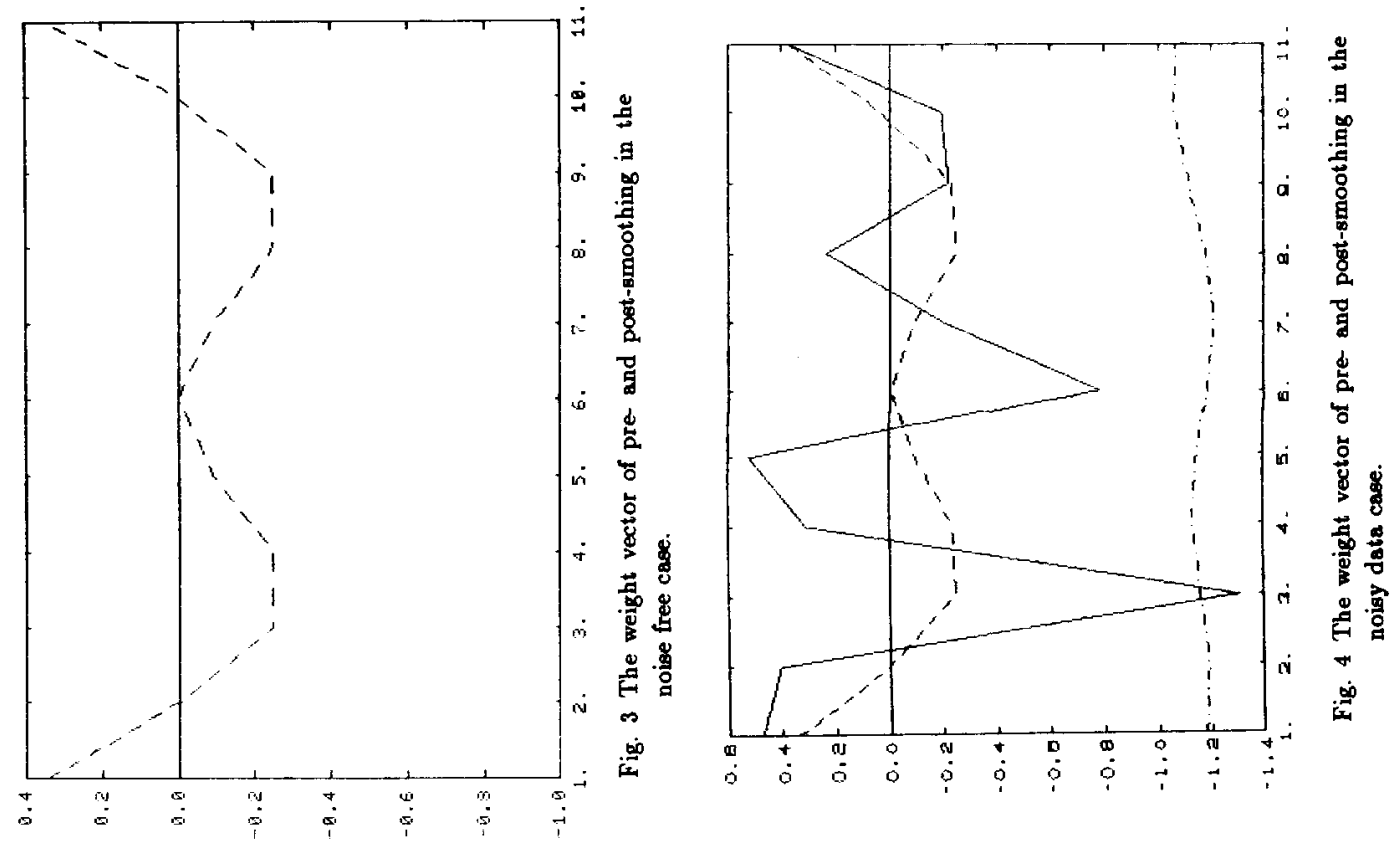

Fig. 5 Zero diagram of the LSE weight vector in the noisy dats example, $(x)$ for $\hat{w},(\square)$ for $w_{T O T},(\omega)$ for $\hat{w}$ of the reduced rank data matrix.

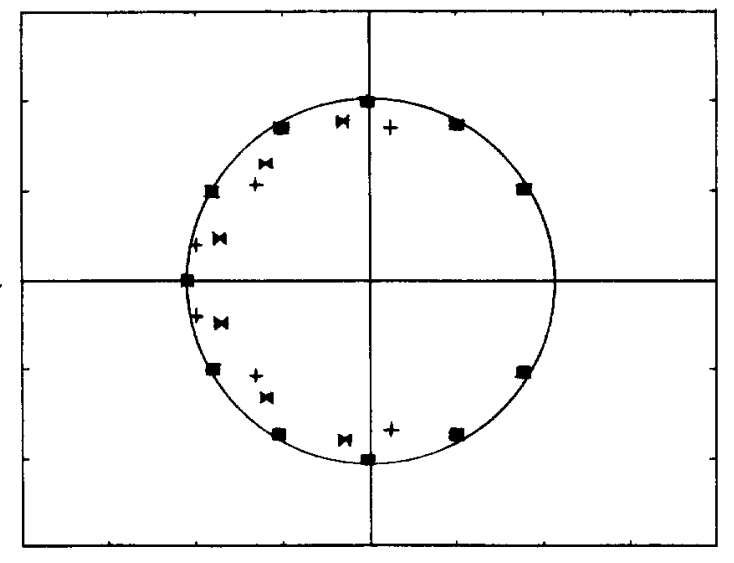

